\title{
Plasma Cleaning and Coating of Sensor Components
}

\author{
Carlotta N. Dietel \\ Diener electronic $\mathrm{GmbH} \&$ Co. KG \\ dietel@plasma.de
}

\section{Summary}

In today's sensor industry finding new environmentally friendly and efficient methods for surface treatment in order to achieve clean and functionalized surfaces has become absolutely essential.

One of these methods has proven to be low pressure and atmospheric pressure plasma treatment. Unlike traditional chemical methods plasma treatment allows for immediate further processing of the samples so that the most effective use of the surface treatment can be made, leading to better bonding and adhesion. During plasma treatment the surface is first of all cleaned of all organic contaminants, oil-residues and oxides and is then activated and polarized creating radical-sites which form a sturdy and lasting bond. Even very difficult oxide films can be removed with this plasma technology.

Using this same technology these surfaces can also be nano-coated via plasma polymerization. This allows a functionalization of the surface to take place in order to achieve various different surface properties such as hydrophilicity, hydrophobicity, corrosion protection, isolating layers etc.

Low pressure and atmospheric pressure plasma technology (Diener electronic Gmbh \& Co KG) is already being used by many large and small companies around the world in $R \& D$ and in series manufacturing.

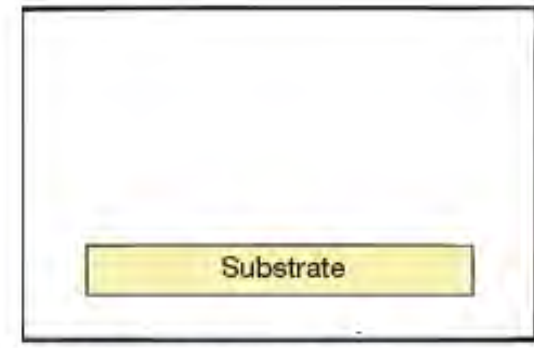

fig. 51: before plasma treatment

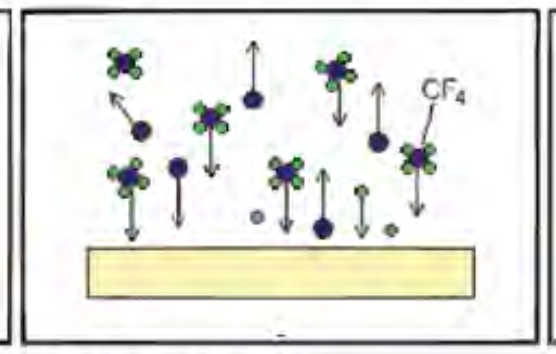

fig. 52: plasma treatment

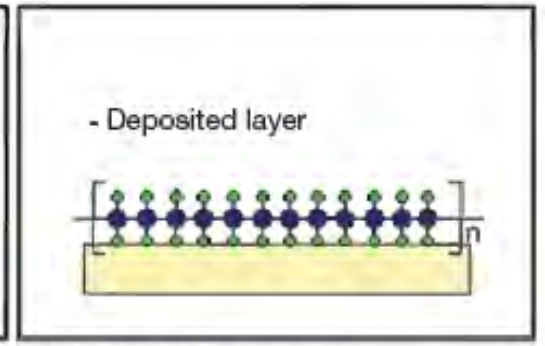

fig. 53: after plasma treatment

Figure 1. Schematic diagram of a plasma polyermisation process

\section{Process technology}

Plasma is an ionized gas. This means that neutral gas atoms are split into ions and electrons. Energy must be supplied to split neutral gases. This energy is supplied to the plasma with the aid of electrical fields. Direct voltage sources or alternating voltage sources can be used to excite the plasma. Plasma which is generated in the pressure range below approx. 10 mbar is designated as a low pressure plasma.

\section{Coating}

- Monomers can be introduced into the vacuum chamber in order to polymerize in a plasma environment to form thin films on the surface
- Examples

- Adhesion agent coatings

- Barrier coatings

- Teflon-like and hydrophobi coatings

- Corrosion protection

- These coatings can act as primers on the surface of various substrates so that a sufficient amount of polar, unpolar or covalent bonds can be formed between the substrate and the adhesive.

Permanent Hydrophilic Coatings

- At times activation processes can become passive within several hours after treatment. 
- Hydrophilic plasma polymerization coatings allow for coatings thatmaintain these hydrophilic properties on the surface for months and years.

- The coating thickness ranges between $15-200 \mathrm{~nm}$.

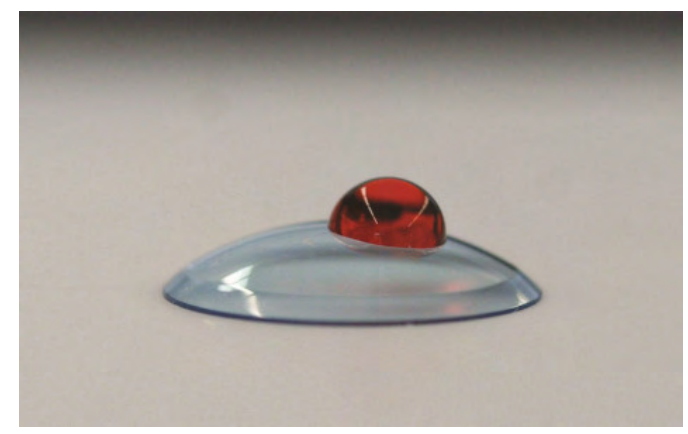

- The permanenct contact angle can be kept below $10^{\circ}$.

- The plasma polymerization coating takes place at low temperatures, thereby not damaging the substrate.

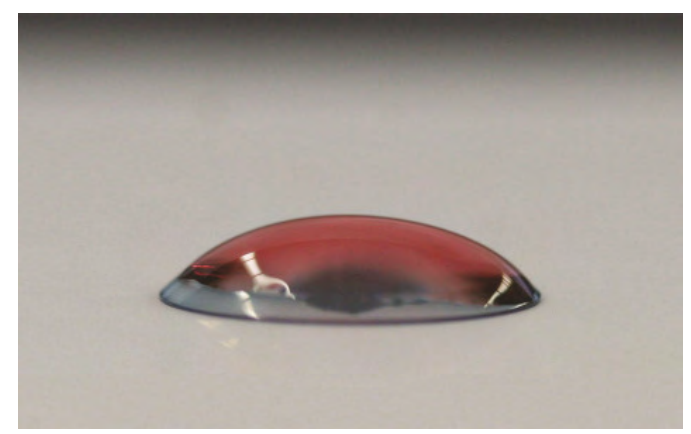

Figure 2: Substrate Before and After Hydrophilic Coating

Hydrophobic Coatings

- There are several types of coating possible including Teflon-like coatings and glass-like coatings.

- These coatings can serve as a protection against water vapor on sensitive parts.

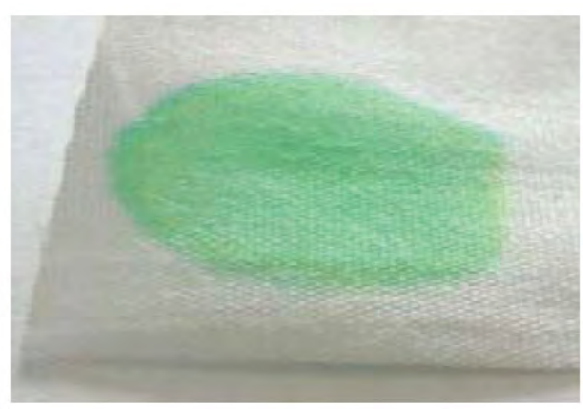

- These coatings are extremely environmentally friendly.

- The coating thickness ranges between $50-$ $500 \mathrm{~nm}$.

- Other properties of the substrate are not influenced by the coating.

- These coatings have been tested and confirmed to be biocompatible (by IGB Fraunhofer Stuttgart).

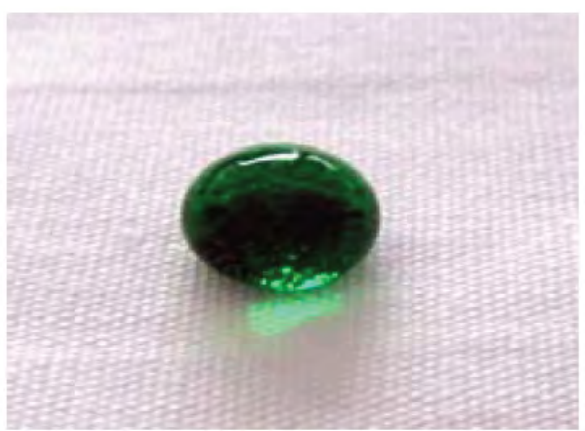

Figure 3: Substrate Before and After Hydrophobic Coating

\section{Conclusion}

The advantages of plasma treatment include the following:

- Effective and reliable processes

- Low running costs

- Environmentally friendly

- Plasma allows for a broad range of surface properties 